\title{
Vidéosurveillance et insécurités urbaines: Etude de l'efficacité préventive du dispositif de caméras installé au quartier des Pâquis à Genève
}

\author{
Raoul Kaenzig and Francisco Klauser \\ Institut de Géographie, Université de Neuchâtel, Espace Louis Agassiz 1, 2000 Neuchâtel, Switzerland \\ Correspondence: Raoul Kaenzig (raoul.kaenzig@unine.ch) \\ and Francisco Klauser (francisco.klauser@unine.ch)
}

Received: 30 August 2017 - Revised: 8 December 2017 - Accepted: 10 December 2017 - Published: 14 February 2018

Résumé. Drawing upon police statistics, this paper explores the impacts of the videosurveillance cameras (CCTV) installed in November 2014 in the Pâquis neighborhood in Geneva. It focuses on the evolution of criminality within the filmed perimeter and nearby settings. This analysis completes a previous article on the perceived safety of residents (Klauser and Kaenzig, 2017). In line with former studies, our results underline the limited preventive effects of the CCTV system. The analysis shows that cameras are inducing criminality displacement, mainly of drug trafficking. Overall, this study provides a differentiated and complex image of CCTV systems as instruments of territorialization of monitored areas.

\section{Introduction}

L'utilisation de caméras de surveillance se généralise et se banalise progressivement. Cette progression est favorisée par l'amélioration de la technologie utilisée ainsi que par un accroissement de la demande sociale en matière de sécurité. Ces dernières années, la sécurité dans l'espace public est en effet devenue un enjeu qui influence fortement le contenu de politiques locales, régionales et nationales (Heilmann et al., 2012). Dans ce contexte, la vidéosurveillance est souvent présentée comme une réponse légitime pour contrer la montée des incivilités et autres problèmes d'insécurité en milieu urbain. En parallèle, s'est développée une littérature scientifique grandissante, relativisant l'efficacité des systèmes de caméras à cet égard (Jeudy, 1990; Fyfe et Bannister, 1998; Williams et al., 2000; Armitage, 2002; Gill et Spriggs, 2005; Welsh et Farmington, 2002).

En Suisse aussi, de nombreux projets de vidéosurveillance ont vu le jour depuis une dizaine d'années (Guéniat et Klauser, 2013), dont celui du quartier des Pâquis à Genève. Ce système comprend vingt-neuf caméras installées durant l'automne 2014 dans un périmètre compris entre la place de Cornavin et les rues du Mont-Blanc, de Berne, Pellegrino-Rossi, De-Monthoux et de Fribourg (cf. « zone pilote », Fig. 1). Il s'agit du dispositif de caméras le plus étendu (spatialement) et avancé (techniquement) de Suisse, focalisé sur des espaces publics extérieurs avec visionnage des images en temps réel. L'investissement lié à son installation s'est élevé à 1.1 millions de francs (Département de la sécurité et de l'économie du Canton de Genève, 2014) et s'inscrit dans le cadre de la mise en œuvre de la convention de lutte contre la criminalité, adoptée conjointement par le Ministère public et le Conseil d'Etat le 29 août 2012 et rééditée le 17 septembre 2014 (République et Canton de Genève, 2014). Les caméras sont pilotées depuis une centrale de visionnage installée dans les locaux de la police cantonale, au Nouvel hôtel de police. Une dizaine de collaborateurs se relaient $20 \mathrm{~h}$ sur $24 \mathrm{~h}$ et 365 jours par an pour visionner les images et diriger les caméras. D'un point de vue opérationnel, le système est aujourd'hui considéré par la police comme un outil supplémentaire, qui nécessite cependant impérativement la présence d'un personnel de surveillance dans la salle de visionnage ainsi que de personnes mobiles prêtes à intervenir sur le terrain ${ }^{1}$.

\footnotetext{
${ }^{1}$ L'évaluation du dispositif réalisée en 2016 a permis de distinguer différents types d'usages en temps réel : (1) les « usages actifs spontanés » des caméras (la surveillance en temps réel, visant à trouver du flagrant délit), (2) les « usages actifs déclenchés » (des opérations en temps réel lancées sur alerte), (3) ainsi que les
} 


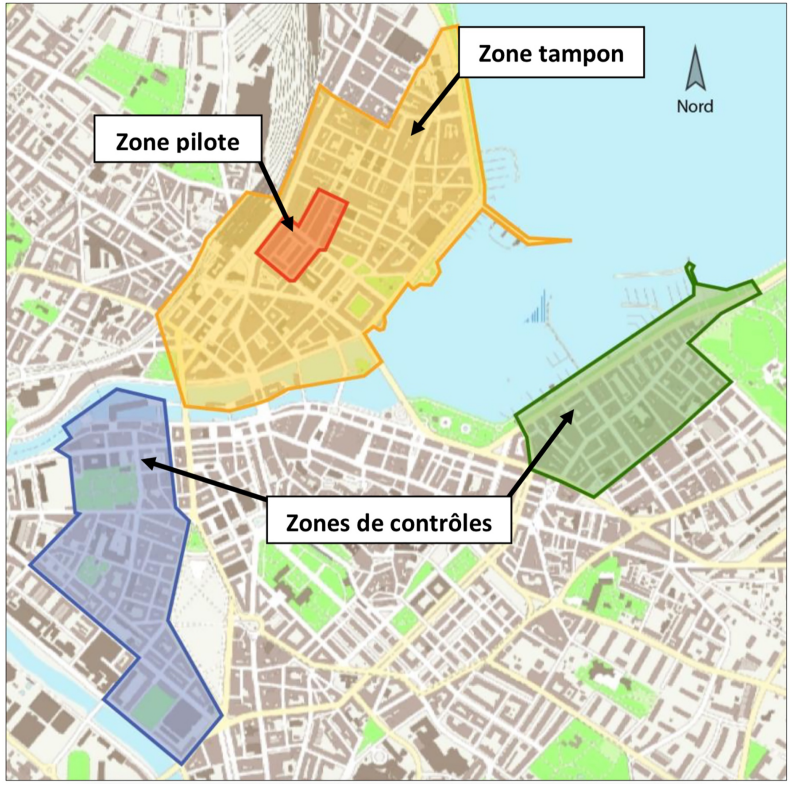

Figure 1. Carte des périmètres retenus pour l'enquête. La zone pilote, dans le quartier des Pâquis, est le périmètre équipé de caméras. Source : SAS Police cantonale de Genève et Institut de géographie UniNe, 2016.

En s'appuyant sur des données statistiques de la police du Canton de Genève, cet article porte sur l'efficacité préventive du système de caméras aux Pâquis ainsi que sur des éventuels effets de déplacement du crime dans d'autres quartiers ou zones avoisinantes ${ }^{2}$. Cette analyse complète un article précédent, rédigé par les mêmes auteurs (Klauser et Kaenzig, 2017), qui adoptait une perspective analytique et méthodologique complémentaire, focalisée sur la manière dont ce dispositif de caméras est vécu et perçu par les habitants du quartier des Pâquis ainsi que par la population genevoise plus généralement.

Les deux articles poursuivent une approche géographique de la vidéosurveillance (Klauser, 2006), visant à mieux comprendre l'efficacité des caméras comme instruments de territorialisation symbolique et informationnelle des espaces télésurveillés. Ci-dessous, nous situons cette approche au sein de la littérature thématique existante, avant de passer aux explications méthodologiques ainsi qu'à l'analyse.

« usages actifs planifiés » (l'usage des caméras à l'occasion d'opérations mises en place de plus longue date) (Kaenzig et Klauser, 2016).

${ }^{2}$ Cette étude a été mandatée par le Département de Sécurité et de l'Economie du Canton de Genève. Elle a été conduite de manière indépendante entre novembre 2014 et novembre 2016 et suivie par un groupe d'accompagnement composé de représentants de la police, de la ville, d'habitants du quartier et de travailleurs-ses du sexe.

\subsection{Effets préventifs et territorialisation symbolique?}

Pour les partisans de la vidéosurveillance, les caméras empêchent préventivement le développement de la criminalité dans les espaces surveillés, tout en améliorant le sentiment de sécurité des «bons » citoyens (Melchior, dans Heilmann et al., 2012). Ces effets préventifs de la vidéosurveillance sont imputés au pouvoir symbolique des caméras, qui agirait sur les qualités sémiotiques des espaces surveillés. La vidéosurveillance pourrait ainsi « informer » les espaces surveillés, leur attribuer des nouvelles significations (p.ex. « cet espace est protégé », « cet espace est plus sûr ») et par là, influencer les usages et les perceptions de ces espaces.

$\mathrm{Si}$, dans ce sens, sont soulignés les bénéfices des caméras en raison de leur pouvoir de territorialisation symbolique des espaces filmés, les opposants à la technologie en rappellent souvent les potentiels effets négatifs en matière d'exclusion sociale (Coleman et Sim, 2000). Dans cette deuxième perspective, la vidéosurveillance est présentée comme un instrument qui disciplinerait les espaces surveillés, en y imposant de manière symbolique un certain ordre social excluant des personnes ou groupes sociaux non conformes à celui-ci ${ }^{3}$.

Pris ensemble, les deux points de vue véhiculent une conception de la vidéosurveillance comme un instrument de pouvoir symbolique, qui projette des informations dans les espaces surveillés et, par là, agit sur la perception et sur les usages de ces espaces. Or, l'essor de la vidéosurveillance a aussi fait émerger une littérature scientifique grandissante, qui permet d'appréhender les limites de cette agentivité symbolique des caméras, et plus spécifiquement la grande complexité du lien entre vidéosurveillance et criminalité (Brown, 1995; Ditton et Short, 1999; Fyfe et Bannister, 1996; Honess et Charman, 1992; Tilley, 1998). Il en ressort que les effets de la vidéosurveillance sur la criminalité ne sont pas aussi manifestes et univoques que ne le suggèrent les fabricants de systèmes de caméras ou autres partisans de cette approche de sécurisation de l'espace (Gill et Spriggs, 2005). S'agissant du cas genevois, une enquête préliminaire à l'installation du dispositif faisait un constat similaire : «... il y a autant d'études qui mettent en évidence un impact positif de la vidéosurveillance sur la criminalité, qu'il y a d'études qui démontrent - au contraire - que la vidéosurveillance n'améliore pas la sécurité. La première conclusion qui s'impose est donc que l'installation de caméras ne suffit pas à faire diminuer la criminalité. En revanche, la vidéosurveillance peut avoir un effet et faire diminuer le nombre de certains délits si un certain nombre de circonstances sont réunies » (SES, $2012: 3$ ).

Plus précisément, trois grands facteurs différenciant l'image de l'efficacité préventive des caméras ressortent du corpus de recherches empiriques menées à ce sujet. Premièrement, la littérature souligne que cette efficacité dépend for-

\footnotetext{
${ }^{3}$ Pour une discussion plus complète de la vidéosurveillance comme outil de territorialisation symbolique et informationnelle des espaces surveillés, voir Klauser et Kaenzig, 2017.
} 
tement du type de criminalité analysé, c'est-à-dire de la nature des délits. La vidéosurveillance s'avère être un système relativement efficace dans le cas de la criminalité rationnelle (qui intègre une réflexion coûts-bénéfices explicite de la part du délinquant) telle que les actes délictueux prémédités (Ditton et Short, 1999). Ce constat explique pourquoi la vidéosurveillance peut effectivement diminuer les vols dans les voitures, dans des parkings placés sous surveillance, par exemple. En revanche, dans le cas de la criminalité affective (émotionnelle ou impulsive), l'efficacité préventive de la vidéosurveillance est faible voire nulle. La vidéosurveillance n'a pratiquement pas d'effets dissuasifs sur les comportements de nature impulsive tels que vandalisme, agressions, bagarres ou troubles de l'ordre public sous l'effet de l'alcool ou de drogues (Brown, 1995; Sivarajasingam et al., 2003).

Deuxièmement, si l'installation de caméras de vidéosurveillance peut contribuer à améliorer la sécurité d'un espace à court terme, et pour certains types de délits, les effets préventifs sur le long terme s'avèrent beaucoup moins concluants. La propension des caméras à dissuader les individus de se livrer à des actes criminels diminue au fil du temps car les délinquants, au même titre que n'importe quel autre usager, tendent à s'accoutumer à la présence des caméras et finissent par les oublier (Armitage, 2002; Cusson, 2006; Gill et Spriggs, 2005; Welsh et Farmington, 2002).

Finalement, les études empiriques suggèrent que les effets de la vidéosurveillance ne sont pas seulement limités dans le temps mais aussi dans l'espace. L'efficacité de la vidéosurveillance est géographiquement limitée et peut avoir pour effet de déplacer la criminalité dans les zones dépourvues de caméras (Brown, 1995; Cameron et al., 2008; Gill et Spriggs, 2005; Tilley, 1998; Welsh et Farrington, 2002). Ce déplacement de la criminalité lié à la présence d'un dispositif de caméras de surveillance est couramment nommé « effet plumeau » (Bowers et Johnson, 2003; Skinns, 1998; Tilley, 1998) et sera analysé dans le présent article en référence aux infractions liées au trafic des stupéfiants notamment.

Le présent article contribue à cette littérature en proposant une étude de cas unique à plusieurs égards : il s'agit nonseulement de la seule étude de cette nature qui a été réalisée en Suisse, mais aussi de l'une des rares recherches - à une échelle internationale - portant sur une durée d'évaluation aussi étendue (deux années d'évaluation depuis la mise en fonction des caméras). L'intérêt particulier de ce cas réside également dans les caractéristiques spécifiques de la zone surveillée; ce quartier à forte mixité sociale situé en plein centre-ville se caractérise par des usages très variés. Ses rues sont autant fréquentées par les touristes séjournant dans les hôtels de luxe, par les nombreux résident-e-s qui y vivent, par les enfants qui fréquentent les écoles du quartier ainsi que par les noctambules qui le considèrent comme l'un des foyers de la vie nocturne de la cité. En parallèle, les Pâquis sont également connus pour abriter une forte densité d'activités liées à la prostitution ainsi que pour le trafic de stupéfiants. De cette mixité sociale découlent des enjeux im- portants en matière de pouvoir et de conflits d'usages potentiels, qui renforcent la pertinence de notre étude. Par ailleurs, l'installation des vingt-neuf caméras a connu un très fort retentissement médiatique - amplifié encore par le caractère sensible du thème de la prostitution - et déclenché de vives réactions citoyennes (Roselli, 2014). Ce fort écho médiatique est susceptible d'avoir contribué, pour le moins dans un premier temps, à une visibilité symbolique accrue des caméras avec comme effet une efficacité préventive renforcée. Notre étude est ainsi particulièrement adaptée pour analyser l'évolution du pouvoir de territorialisation symbolique inhérent à la vidéosurveillance à travers le temps. Finalement, la qualité des statistiques policières à disposition permet d'analyser de manière particulièrement fine et rigoureuse l'évolution de différents types de criminalité, ce qui fait de ce cas d'étude un terrain privilégié aussi d'un point de vue empirique. Les données mobilisées dans cet article sont présentées avec plus de détails dans la partie suivante.

\section{Méthodologie}

Notre analyse repose sur les statistiques policières du service d'analyse stratégique $\left(\mathrm{SAS}^{4}\right)$ de la police cantonale de Genève. Ces statistiques permettent de mesurer l'évolution de différents types de criminalité ${ }^{5}$ dans des zones géographiques différentes, ici zone pilote, zone tampon et zone de contrôle. Les statistiques policières recensent toutes les interpellations de la police genevoise, indépendamment de la méthode utilisée (avec l'aide ou non des caméras). En outre, cette base de données répertoriant tous les contrôles et interventions de la police (permettant ainsi de mesurer l'évolution de la présence policière au quartier des Pâquis), nous permet d'évaluer dans quelle mesure les variations des infractions sont corrélées, ou non, à l'activité policière sur le terrain. Pour cet article nous avons travaillé sur les données allant de janvier 2011 à août 2016.

Malgré la richesse de cette source d'informations, il est à noter que la mesure de la criminalité est toujours partielle. Les statistiques de la criminalité ne recensent, par définition, que les cas connus de la police. Il reste donc une part de criminalité non connue des autorités et donc non quantifiable (Killias et al., 2011). Par ailleurs, « la mesure de la criminalité dépend du personnel, des priorités et de l'efficacité de la police et de la justice, et de la propension de la population à dénoncer les actes délictueux. Il est en général difficile de

\footnotetext{
${ }^{4}$ Ce service est chargé de suivre l'évolution des phénomènes sociaux et criminels pouvant influencer le contexte dans lequel la police doit intervenir.

${ }^{5}$ D'un point de vue juridique, les termes relatifs à « criminalité » et « délinquance » renvoient à des peines différentes. Dans cet article, ces deux notions sont utilisées indistinctement pour mentionner des activités ayant mené à des infractions pénales. La criminalité et la délinquance sont mesurées par le nombre d'infractions au Code pénal suisse.
} 
déterminer les facteurs à l'origine des variations enregistrées en matière de criminalité. » (OFS, 04.08.17, en ligne) ${ }^{6}$.

Dans notre enquête, nous avons travaillé par triangulation méthodologique, en couplant les méthodes quantitatives avec d'autres méthodes (questionnaires, entretiens, observations et parcours commentés) afin d'obtenir un faisceau de données nous permettant d'affiner les tendances quantitatives. Le présent article se base principalement sur les résultats issus des statistiques policières, tout en corroborant ceux-ci avec les autres approches méthodologiques mobilisées lors des recherches précédentes (Kaenzig et Klauser, 2016).

Pour les besoins de cette étude, une sélection d'indicateurs a été déterminée afin d'évaluer de manière quantitative les effets préventifs du système de vidéosurveillance à Genève. Les lignes suivantes décrivent les indicateurs retenus selon trois axes distincts : les indicateurs de la criminalité, les indicateurs par types de criminalité et finalement les indicateurs sur les stupéfiants.

\subsection{Les indicateurs de la criminalité}

De manière générale, nous avons distingué deux familles d'indicateurs pour appréhender les variations de la criminalité : les infractions sur la voie publique et les infractions totales. D'abord, nous avons mobilisé une sélection d'infractions regroupant l'ensemble des délits commis sur la voie publique tels que vols, agressions, infractions à la circulation et dommages à la propriété. Il s'agit des infractions pour lesquelles la vidéosurveillance pourrait, selon la littérature scientifique existante (Fonteneau, 2008), avoir un effet puisque les caméras ne filment que les espaces publics de la zone vidéosurveillée.

Considérant que ces variables sont susceptibles d'être influencées par la mise en service des caméras, cette première famille d'indicateurs est ensuite comparée aux infractions totales qui regroupent la totalité des délits répertoriés par la police, soit toutes les infractions au Code pénal suisse. La comparaison entre les infractions sur la voie publique et les infractions totales permet ainsi de s'assurer que les tendances observées ne sont pas uniquement imputables au choix des infractions retenues pour l'étude.

Hormis ce premier regard portant sur les indicateurs susmentionnés, notre analyse s'intéresse également à l'efficacité de la vidéosurveillance selon des types de criminalité plus spécifiques.

\subsection{Les indicateurs par types de criminalité}

Pour appréhender les effets des caméras sur les différents types de criminalité, nous distinguons deux groupes d'infractions : la criminalité rationnelle et la criminalité affective. Pour la première catégorie ont été retenues les infractions qui intègrent une réflexion coûts-bénéfices explicite de

\footnotetext{
${ }^{6}$ httplunhboxlvoidb@x portal/fr/index/themen/19/01/pan.html, consulté le 04.08.2017.
}

la part des délinquant-e-s ${ }^{7}$, alors que la deuxième contient toutes les infractions de nature impulsive ou émotionnelle ${ }^{8}$. Respectivement, selon la littérature scientifique, les dispositifs de vidéosurveillance sont d'une certaine efficacité pour la première catégorie alors que cette efficacité est faible voire nulle pour la seconde.

Il est certes difficile de définir la nature exacte d'une infraction, mais ces catégorisations permettent néanmoins de distinguer les effets des caméras sur des types de criminalité distincts. Dans notre cas précis, ils ont par contre l'inconvénient de se fonder sur des volumes d'événements relativement faibles, ce qui invite à interpréter les résultats avec prudence. Nous pouvons également souligner que les infractions en lien avec des agressions ou encore des conflits personnels n'ont pas été retenues dans cette catégorisation, car il est impossible de discerner la part de préméditation ou d'impulsivité inhérente à ces situations. Finalement, nous avons aussi retenu et analysé de manière plus spécifique l'évolution des infractions liées au trafic des stupéfiants, ce qui nécessite des explications méthodologiques plus détaillées.

\subsection{Les indicateurs liés aux stupéfiants}

Les statistiques sur les stupéfiants englobent toutes les infractions à la loi sur les stupéfiants ${ }^{9}$ (LStup, Loi fédérale sur les stupéfiants et les substances psychotropes), à l'exception de l'infraction «possession de stupéfiants ». Cette dernière catégorie n'est pas incluse dans notre analyse car notre intérêt porte ici sur le trafic de stupéfiants, soit l'effet des caméras concernant les transactions, potentiellement filmées, entre vendeur(s) et acheteur(s) de drogues. Ces données statistiques sur les stupéfiants sont également à interpréter avec précaution car il est difficile de savoir si elles reflètent l'intensité de l'activité policière (augmentation ou diminution des contrôles) ou celle de l'activité liée au trafic de drogue (augmentation ou diminution du trafic). Toutefois, les variables de contrôle ainsi que les approches méthodologiques complémentaires nous permettent de valider avec plus de certitude nos résultats à cet égard.

\section{Analyse}

Cette partie consacrée aux résultats est structurée en trois axes, suivant l'ordre de présentation des indicateurs de la

\footnotetext{
${ }^{7}$ Liste des infractions retenues pour la catégorie rationnelle : cambriolage (commerce, magasins, administration, entreprise, pharmacie, laboratoire, cabinet médical, cafés/restaurants), vol (à l'astuce, sur/dans un véhicule, à l'arraché, de véhicule).

${ }^{8}$ Liste des infractions retenues pour la catégorie affective : moeurs (exhibitionniste), incendie (intentionnel, intentionnel, de véhicules), dommages à la propriété (sur la voie/lieu public, dans un bâtiment public, tag/graffiti, sur des véhicules).

${ }^{9}$ Catégories d'infractions à la loi sur les stupéfiants : trafic, culture, consommation, exportation et importation.
} 


\section{Evolution du nombre d'infractions totales par mois dans la zone pilote : 2011-2016}

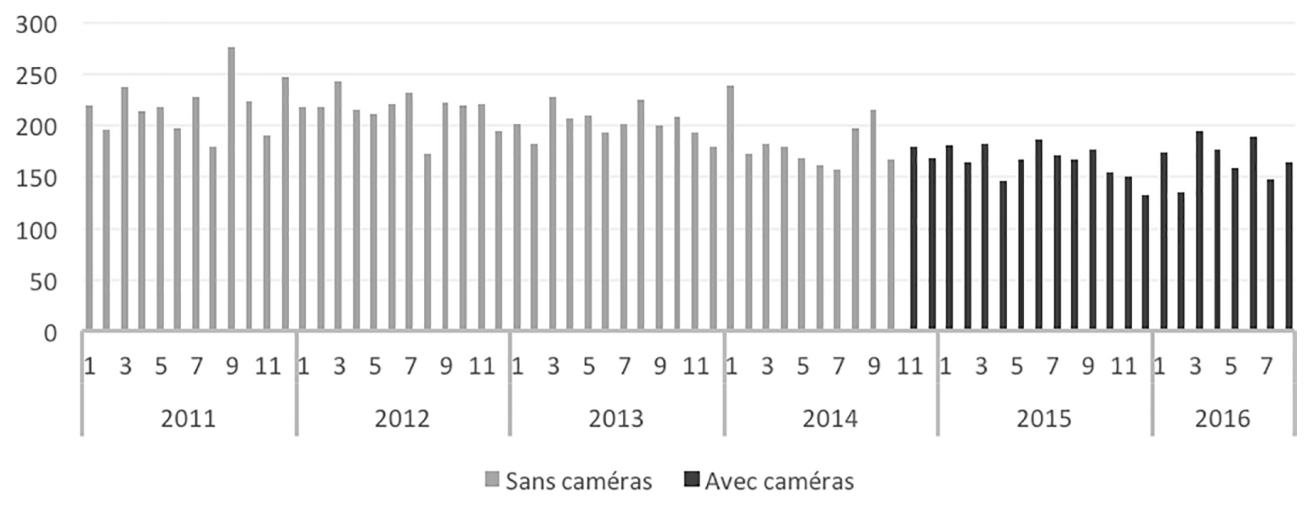

Figure 2. Nombre d'infractions totales par mois dans la zone pilote. La période sans caméras est en gris et celle avec caméras en noir (Source : SAS, monitoring 2016).

criminalité du chapitre précédent. Premièrement, nous abordons l'évolution de la criminalité aux Pâquis en général, en distinguant les infractions sur la voie publique et les infractions totales. Deuxièmement, nous étudions les effets de la vidéosurveillance sur la criminalité rationnelle, puis affective Finalement, nous traitons la question des effets de déplacement de la criminalité induits par les caméras, en référence notamment à la problématique du trafic de stupéfiants.

\section{1 Évolution générale de la criminalité}

Les statistiques policières génèrent une image précise de l'évolution de la criminalité dans la zone pilote. Les graphiques suivants démontrent l'évolution de la totalité des infractions (Fig. 2) et des infractions sur la voie publique (Fig. 3). Nous constatons pour les deux types de données une tendance à la baisse lors de la période précédant l'installation des caméras, soit de janvier 2011 à octobre 2014. En contraste, à partir de la période 2015-2016, ces tendances soit s'atténuent, soit s'inversent.

Concernant les infractions totales (Fig. 2), la période avec caméras se caractérise donc par une continuation de la baisse observée lors de l'époque sans caméras, pourtant bien moins marquée. Dans le cas des infractions sur la voie publique (Fig. 3), pour lesquelles la vidéosurveillance aurait pu avoir un effet, on observe par contre une légère augmentation de la criminalité depuis l'installation des caméras.

Cette légère hausse du nombre d'infractions répertoriées sur la voie publique pourrait s'expliquer par une augmentation des interpellations policières. Or, les statistiques à disposition montrent qu'il n'y a pas eu plus d'interpellations policières dans cette zone à cette période. Une autre hypothèse consisterait à postuler une meilleure efficacité du travail policier grâce aux caméras. Toutefois, cette hypothèse est également à rejeter car nous observons en effet une image plus complexe, avec aussi certains types de délits à la baisse (cf. partie suivante). Restons ainsi au constat simple qu'aucun effet préventif ne peut être constaté à ce premier niveau d'analyse.

En prolongeant cette réflexion, le tableau suivant (Tableau 1) contient les variations interannuelles précises des infractions sur la voie publique et des infractions totales, en distinguant les trois zones étudiées dans notre enquête (zone pilote, zone tampon et zone de contrôle). Là encore, force est de constater la baisse marquée des infractions entre 2011 et 2014. D'une part, concernant les variations des infractions totales, la baisse la plus importante dans la zone pilote a eu lieu de 2013 à 2014 (-12\%). Les années suivantes se caractérisent par une diminution plus modérée de $-6 \%$ (20142015) et de $-2 \%$ (2015-2016). D'autre part, concernant les infractions sur la voie publique, la baisse la plus importante dans la zone pilote a eu lieu entre 2012 et $2013(-24 \%)$. Ensuite, elle connaît une hausse de $15 \%$ entre 2015 et 2016 . A noter aussi, de manière générale, que le faible volume de données pour les infractions sur la voie publique nous invite à ne pas surinterpréter cette légère tendance à la hausse, observée depuis l'installation des caméras. Nous retenons surtout que les statistiques mobilisées ne permettent pas d'identifier un quelconque effet préventif des caméras sur les variations de la criminalité.

La partie suivante apporte un élément supplémentaire à cette analyse, en distinguant la criminalité rationnelle de la criminalité affective, ce qui nous permettra de confronter notre enquête aux résultats de la littérature existante. Cette dernière fait état d'une certaine efficacité des caméras s'agissant du premier type de criminalité mais non pas du deuxième.

\subsection{Criminalité rationnelle versus criminalité affective}

Le graphique suivant montre que depuis la fin de l'année 2011, la criminalité rationnelle tend à diminuer dans la zone 


\section{Evolution du nombre d'infractions sur la voie publique par mois dans la zone pilote : 2011-2016}

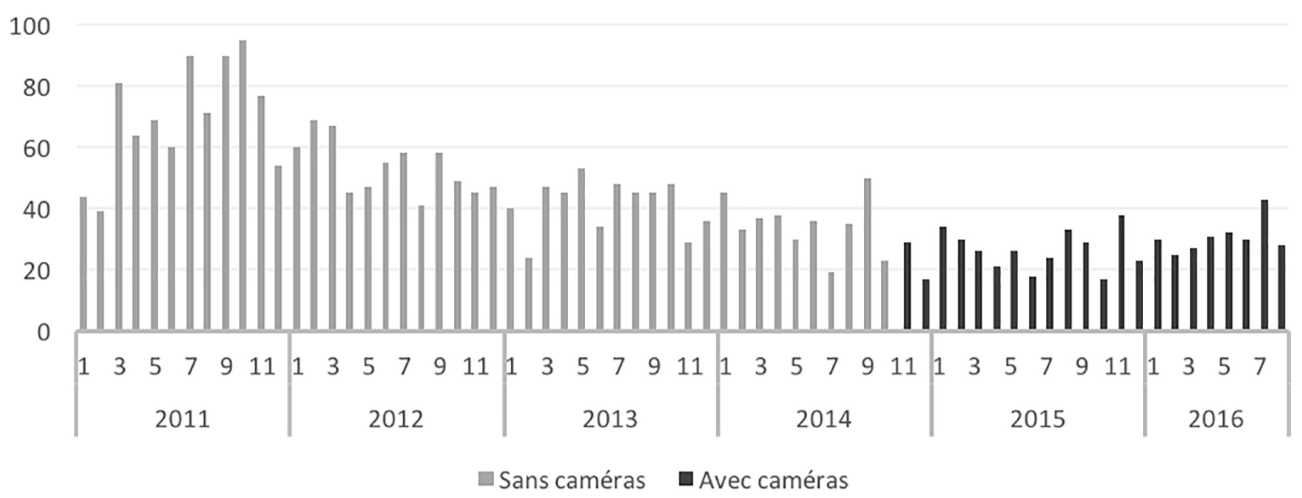

Figure 3. Nombre d'infractions sur la voie publique par mois dans la zone pilote. La période sans caméras est en gris et celle avec caméras en noir (Source : SAS, monitoring 2016).

Tableau 1. Tableau illustrant les variations interannuelles des infractions (infractions sur la voie publique et total des infractions) au cours des huit premiers mois de 2011 à 2016 (Source : SAS, monitoring 2016).

\begin{tabular}{lrrr|rrr}
\hline \multirow{2}{*}{$\begin{array}{l}\text { Variations } \\
\text { interannuelles }\end{array}$} & \multicolumn{2}{c}{ Infractions sur la voie publique } & \multicolumn{2}{c}{ Total des infractions } \\
\cline { 2 - 6 } & Zone pilote & Zone tampon & Zone de contrôle & Zone pilote & Zone tampon & Zone de contrôle \\
\hline $2011-2012$ & $-15 \%$ & $-16 \%$ & $-18 \%$ & $+2 \%$ & $-3 \%$ & $-1 \%$ \\
$2012-2013$ & $-24 \%$ & $-17 \%$ & $-13 \%$ & $-5 \%$ & $-19 \%$ & $-10 \%$ \\
$2013-2014$ & $-19 \%$ & $-27 \%$ & $-17 \%$ & $-12 \%$ & $-10 \%$ & $-17 \%$ \\
$2014-2015$ & $-22 \%$ & $0 \%$ & $-1 \%$ & $-6 \%$ & $-3 \%$ & $+3 \%$ \\
$2015-2016$ & $+15 \%$ & $-2 \%$ & $-10 \%$ & $-2 \%$ & $-11 \%$ & $-15 \%$ \\
\hline
\end{tabular}

pilote, malgré de fortes fluctuations mensuelles (Fig. 4). Cette diminution se poursuit également lors de la période suivant l'installation des caméras, ce qui complexifie la tendance générale observée auparavant concernant les délits sur la voie publique.

Quant à la criminalité affective, il est difficile d'y déceler une tendance particulière, si ce n'est des variations plus ou moins marquées selon les périodes prises en compte. Depuis l'installation des caméras, c'est donc principalement la criminalité rationnelle qui continue de baisser alors qu'aucun changement particulier ne peut être souligné s'agissant de la criminalité affective.

Pour rappel, les volumes des événements pour ces données sont très faibles. Par conséquent, les courbes sont à interpréter avec prudence. Par contre, afin de compléter la présente analyse, les statistiques policières peuvent être discutées à la lumière des résultats obtenus par les deux enquêtes par questionnaire réalisées en 2015 et en 2016 auprès des habitants-es de la zone vidéosurveillée ainsi que dans les quartiers alentours. Cette démarche permet d'évaluer dans quelle mesure les tendances statistiquement mesurées sont également vécues et observées par les habitants-es et les usagers-ères des Pâquis. Les résultats ainsi obtenus laissent apparaître que, selon les répondants-es, le bruit, le trafic de stupéfiants et les incivilités n'ont pas diminué suite à l'installation des caméras. En revanche, aucune tendance claire ne se dégage des réponses concernant la perception des habitants-es quant aux actes de violence et aux vols. Les taux de «Ne sais pas/Sans opinion » sont très importants pour ces dernières catégories et cela peut s'expliquer par la difficulté à estimer l'évolution d'événements de ce type sans en être directement la/le témoin (Klauser et Kaenzig, 2017).

Ces résultats confirment l'inexistence d'effets préventifs de la vidéosurveillance sur la criminalité au quartier des Pâquis, comme cela a été démontré dans d'autres cas (Guéniat et Klauser, 2013). De ce point de vue, le dispositif de caméras n'a donc pas reterritorialisé les espaces filmés. Cependant, notre analyse doit être complétée en questionnant les déplacements potentiellement induits par la présence de ce nouveau système de contrôle de l'espace public.

\subsection{Déplacements de la criminalité}

Malgré le peu de changements en matière de criminalité pouvant être attribués aux caméras dans le quartier étudié, le risque inhérent à la pose de tels dispositifs est de provoquer un déplacement de certaines activités criminelles vers des périmètres non-filmés. Ce phénomène, aussi appelé «l'effet 


\section{Evolution de la criminalité rationnelle et affective dans la zone pilote : 2011-2016}

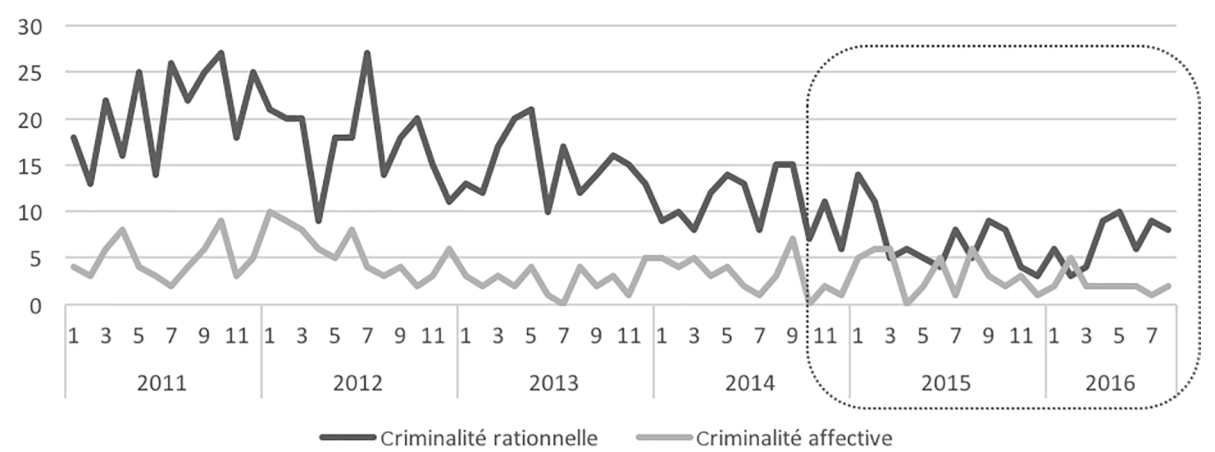

Figure 4. Nombre d'infractions répertoriées par mois dans la zone pilote par types de criminalité. Le rectangle en pointillé met en évidence la période de mise en service des caméras (Source : SAS, monitoring 2016).

plumeau », a été démontré de manière constante dans la littérature scientifique (Carli, 2009; Brown, 1995; Gill et Spriggs, 2005; Tilley, 1998; Waples et al., 2009; Welsh et Farrington, 2002; Waples et al., 2009).

Pour identifier d'éventuels phénomènes de déplacement, nous avons d'abord retenu dans notre analyse l'ensemble des types de délits appartenant à la catégorie de la criminalité rationnelle. Il est en effet reconnu que cette catégorie de délits est la plus à même de se produire ailleurs que dans la zone vidéosurveillée, puisque les délinquants peuvent considérer la présence des caméras dans leur « pesée des intérêts » relative à la réalisation de l'acte délictueux. Cependant, nos résultats ne démontrent ni une baisse significative de la criminalité rationnelle dans le centre filmé des Pâquis (zone pilote), ni une hausse dans les rues voisines (zone tampon) ou dans les zones de contrôle situées dans d'autres quartiers de la ville (Fig. 5). L'interprétation de ces données ne permet donc pas de conclure à un déplacement de la criminalité au sein des zones étudiées.

Ces tendances sont par ailleurs corroborées par les résultats des enquêtes par questionnaire. A titre d'exemple, $58 \%$ des répondants de l'enquête de $2016^{10}$ estiment que les caméras ont eu pour effet de déplacer certains types de criminalité et parmi ces répondants, plus du $80 \%$ estiment que ce sont les activités liées au trafic de drogue qui se sont déplacées (Kaenzig et Klauser, 2016). La partie suivante approfondit donc la thématique spécifique du trafic de drogue en mobilisant les données statistiques policières disponibles.

\subsection{Déplacement du trafic de stupéfiants}

Avant l'installation des caméras dans le quartier des Pâquis, la crainte des déplacements du deal avait été l'une des préoccupations centrales des associations citoyennes et des médias

\footnotetext{
${ }^{10}$ Pour avoir l'opinion des habitants-es et des usagers-ères réguliers-ères du quartier des Pâquis, ne sont considérés que les répondants qui fréquentent le quartier au moins deux fois par mois.
}

régionaux qui relayaient également le scepticisme de certains élus à l'égard de la vidéosurveillance (Armanios, 2013; Languin, 2013). L'intérêt public porté à cette problématique légitime d'autant plus notre démarche visant à mesurer les conséquences spatiales induites par la présence de ce dispositif de surveillance.

Dans la zone pilote, une tendance à la diminution des infractions sur les stupéfiants peut être observée pour la période 2014-2016 (Fig. 6). Cette tendance se poursuit depuis la mise en marche des caméras en octobre 2014, avec néanmoins des pics plus élevés comme en janvier 2015 ou avril 2016. A l'inverse, il est à noter qu'entre octobre 2014 et avril 2015, les infractions ont fortement augmenté dans les quartiers limitrophes (zone tampon) et dans les quartiers de contrôle. Ce pic correspond à la période de l'annonce publique de la mise en marche du dispositif de vidéosurveillance. Toutefois, cette augmentation retombe quelques mois plus tard et fluctue dès lors de mois en mois. Ce fléchissement doit être attribué à l'opération policière spéciale de lutte contre le trafic de stupéfiants ${ }^{11}$ ayant démarré à cette période.

De manière générale, les courbes de tendance de la zone pilote et de la zone tampon sont parallèles et décrivent les deux une tendance à la baisse. Ainsi, dans le domaine des stupéfiants, on constate que si un certain « effet plumeau » peut effectivement être observé, celui-ci se caractérise par des déplacements de très courtes distances (rues voisines) et de courtes durées, particulièrement marqué au début de la mise en service des caméras. Par ailleurs, en ne retenant que les cas d'infractions aux stupéfiants concernant le trafic, c'est-à-dire les transactions, les tendances sont parfaitement similaires et soutiennent également l'hypothèse d'un déplacement des transactions de stupéfiants en marge de la zone filmée.

\footnotetext{
${ }^{11}$ Opération coordonnée de la police pour lutter contre le trafic de stupéfiants : en cours depuis avril 2015.
} 


\section{Evolution de la criminalité de type "rationnelle" dans les trois zones de l'étude : 2014-2016}

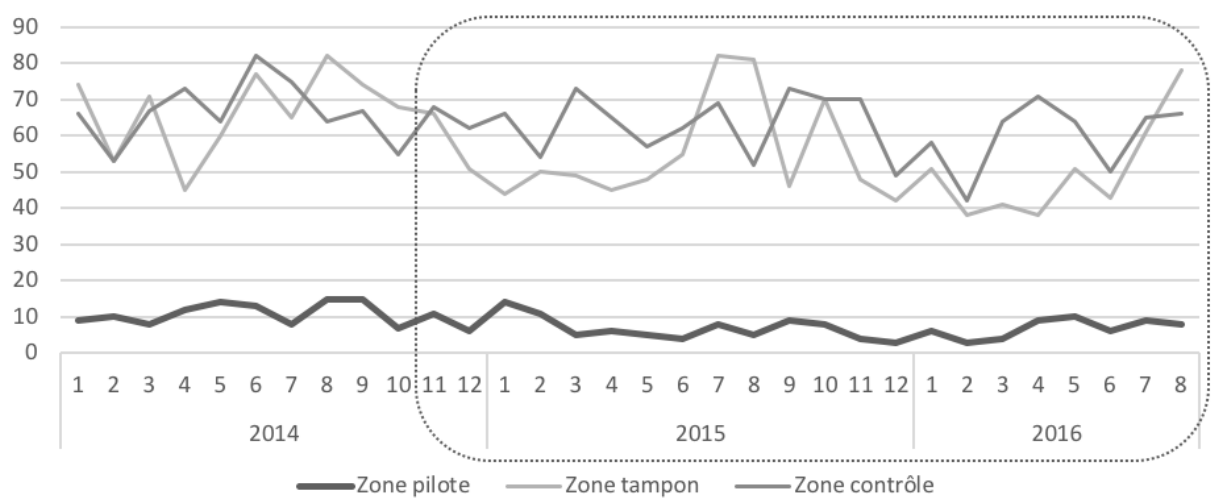

Figure 5. Nombre de cas répertoriés relevant de la criminalité rationnelle dans les trois zones de l'étude lors des deux dernières années. Le rectangle en pointillé met évidence la période de mise en service des caméras (Source : SAS, monitoring 2016).

\section{Infractions à la loi sur les stupéfiants dans les trois zones de l'étude : 2014-2016}

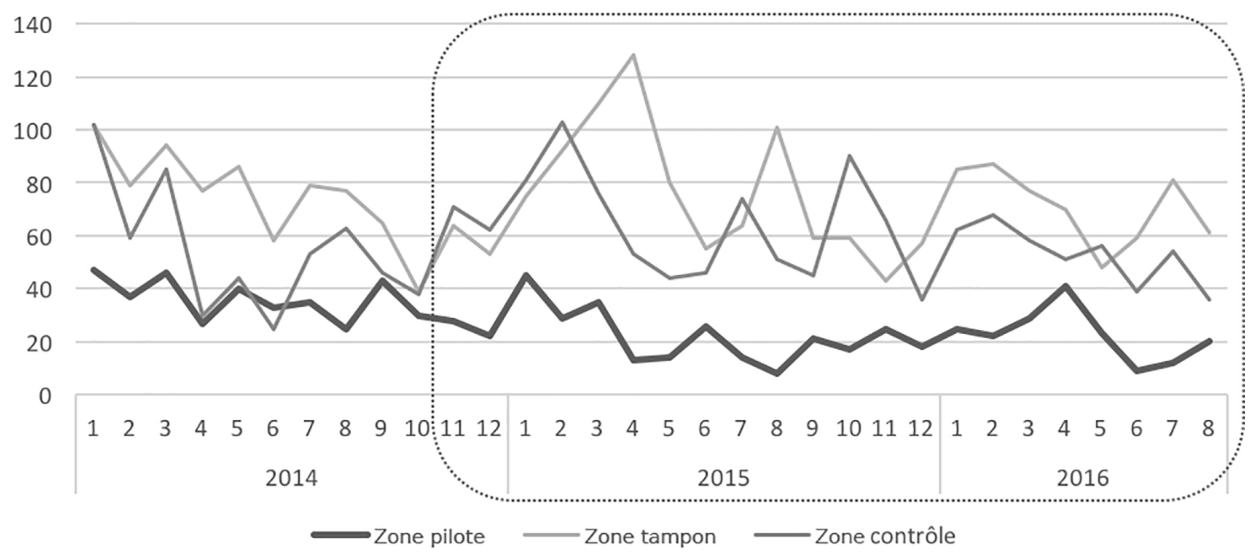

Figure 6. Evolution des infractions à la loi sur les stupéfiants pour les trois zones de l'étude. Le rectangle en pointillé met en évidence la période de mise en service des caméras (Source : SAS, monitoring 2016). (Les infractions à la loi fédérale sur les stupéfiants (Lstup) concernent les cas de trafic (transaction), de culture, de consommation ainsi que d'exportation et d'importation.)

Cet éclatement des zones de trafic aux alentours du périmètre filmé peut également être apprécié en s'intéressant aux données géolocalisées des infractions se rapportant au trafic. Les trois cartes produites ci-dessous illustrent l'évolution spatiale du trafic de stupéfiants sur les huit premiers mois ${ }^{12}$ des années 2014, 2015 et 2016 (Fig. 7). Si les transactions n'ont pas disparu de la zone équipée de caméras, les cas localisés dans la zone tampon sont cependant de plus en plus nombreux et les transactions se font sur un territoire plus étendu qu'en 2014.

\footnotetext{
${ }^{12}$ Les données de 2016 s'étendent de janvier à août. Pour pouvoir comparer cette année avec les autres, nous avons donc retenu les huit premiers mois des trois années d'observation.
}

Notons aussi que cette image statistique d'un déplacement du trafic de stupéfiants correspond à la fois à l'avis de la population large $-54 \%$ des participants à notre enquête par questionnaire en 2016 avaient cet avis - tout comme aux témoignages approfondis de personnes directement concernées, recueillis dans notre enquête à travers quarante-six entretiens semi-directifs avec des habitants-es, commerçantses, travailleurs-ses du sexe, autres usagers-ères du quartier, policiers-ères, et opérateurs du système. Outre cet « effet plumeau » de proximité, les personnes interviewées évoquent également une forte adaptation des pratiques concernant le trafic. Les habitants-es et les commerçants-es du quartier notamment se sont plaints de l'usage de plus en plus fréquent des entrées de bâtiments et des escaliers d'immeubles par les 

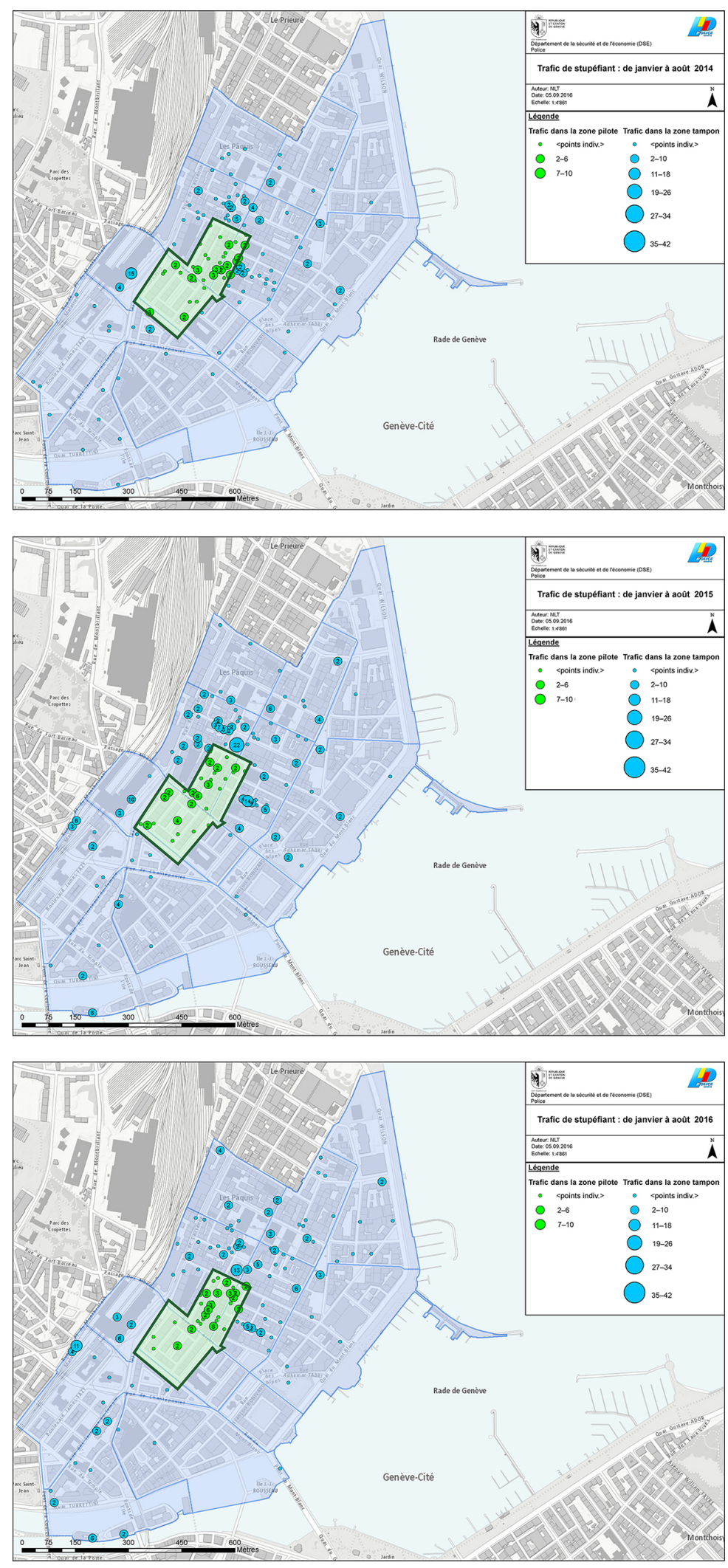

Figure 7. Cartes du trafic de stupéfiants sur les huit premiers mois de 2014, 2015 et 2016 dans la zone pilote de l'étude (en vert) et dans la zone tampon (en bleu). La taille des cercles est proportionnelle au nombre de cas de trafic enregistrés par la police (Source : SAS, monitoring 2016). 
vendeurs et les acheteurs qui y effectuent leurs transactions. La police cantonale, quant à elle, a reconnu que les caméras ont été un élément perturbateur dans les mécanismes des trafiquants de stupéfiants et constate la forte capacité d'adaptation des vendeurs. Suite à l'installation des caméras, ces derniers ont très rapidement modifié leurs pratiques et utilisent de plus en plus des espaces non-filmés tels que les immeubles, des véhicules privés ou certains établissements publics. Cette nouvelle géographie du deal complexifie passablement les interventions de la police qui doit à son tour modifier ses pratiques. C'est à ce niveau que nous observons finalement un vrai effet de territorialisation lié à l'implantation de la vidéosurveillance.

\section{Conclusions}

En résumé, notre analyse apporte un regard nuancé et critique sur le fonctionnement de la vidéosurveillance en tant qu'instrument de territorialisation symbolique. Malgré les discours enthousiastes des concepteurs et des politiques favorables au développement de la vidéosurveillance, notre recherche met en évidence les limites de ce type de dispositif en matière de prévention du crime, coïncidant ainsi généralement avec la littérature scientifique existante. Plus spécifiquement, trois grands enseignements découlant de notre étude de cas méritent d'être soulignés.

Premièrement, les indicateurs utilisés dans notre analyse ne permettent pas de conclure à une baisse des infractions depuis la mise en place des caméras. Une tendance à la diminution générale des infractions a effectivement lieu dans les trois zones de l'étude pilote depuis 2011 jusqu'à fin 2014. Or, depuis la mise en service des caméras, on observe un affaiblissement de cette diminution pour le nombre total des infractions alors que les infractions sur la voie publique pour lesquelles le contrôle des caméras aurait pu avoir un effet - sont caractérisées par une légère augmentation $(+15 \%$ entre 2015 et 2016).

Deuxièmement, la mesure des différents types de criminalité ne permet pas non-plus d'identifier un effet des caméras sous la forme d'une diminution de la délinquance. Une tendance à la baisse de la criminalité rationnelle peut s'observer depuis 2011 et cette tendance se poursuit, sans ruptures particulières, à la suite de l'installation de la vidéosurveillance. Aucun changement particulier ne peut être souligné s'agissant de la criminalité affective. La portée de ces résultats est en revanche limitée par les faibles volumes de données pour ces catégories respectives.

Troisièmement, notre étude ne mettant pas en évidence une baisse significative de la criminalité dans le centre des Pâquis (zone pilote), ni une hausse dans les rues voisines (zone tampon), on ne peut pas conclure à un déplacement général de la criminalité dans les zones étudiées. Toutefois, la mise en service des caméras a provoqué un « effet plumeau » s'agissant du trafic de stupéfiants. Celui-ci se caractérise par des déplacements de très courtes distances (rues voisines). Les transactions n'ont pas disparu de la zone équipée de caméras, mais les cas localisés dans les rues voisines sont de plus en plus nombreux et les transactions se font sur un territoire plus difficile à contrôler et plus étendu qu'en 2014.

Ces résultats portent sur les effets des caméras en matière de prévention et, par conséquent, de déplacements, et viennent compléter un premier article qui soulignait que, du point de vue des habitants-es et des usagers-ères du quartier, la vidéosurveillance n'avait pas redessiné la dynamique du quartier, notamment du point de vue de sa fréquentation, et était relativement bien acceptée par la population. Toutefois peu enthousiastes et peinant à en percevoir les réels bénéfices, les personnes interrogées tendent à banaliser, voire à oublier la présence de ce dispositif sécuritaire. Or, l'analyse des statistiques policières nous permet de compléter ces premières observations et d'observer que, si les caméras ne bouleversent pas la dynamique du quartier, elles semblent tout de même modifier les pratiques s'agissant du trafic de stupéfiants.

Ce dernier aspect appelle un prolongement de la discussion quant à la manière de saisir la complexité des phénomènes de déplacements.

Notre étude permet de souligner que la spatialité des déplacements ne saurait se réduire à l'expression consacrée de « l'effet plumeau ». En effet, si l'on observe à Genève un éclatement des zones de trafic de stupéfiants, il s'agit essentiellement de micro-déplacements. Il serait donc pertinent d'analyser plus en profondeur ces réajustements spatiaux en se penchant sur la capacité d'adaptation des acteurs-trices liés-ées aux activités du trafic. L'installation des caméras a en effet très rapidement été perçu comme un élément venant perturber les pratiques usuelles qui se déroulaient au centre des Pâquis. Très rapidement, vendeurs et acheteurs ont pris la mesure de cette nouvelle configuration en concrétisant leurs transactions dans des espaces qui échappent au regard verticalisé des caméras de surveillance. Ces stratégies d'évitement ont également eu pour effet de modifier certains modes opératoires de la police qui a dû, elle aussi, se montrer résiliente face aux changements. Ces nouvelles pratiques fondées sur des spatialités informées par la présence de caméras soulignent le pouvoir des dispositifs de vidéosurveillance à restructurer les usages de la ville de certaines catégories de la population. D'un point de vue méthodologique, ces perspectives pavent la voie à des approches qui mettent au premier plan des méthodes ethnographiques privilégiant par exemple des phases d'immersion permettant d'appréhender l'agentivité des acteurs-trices de ce milieu. Dans ce cadre-là, l'investigation pourrait être étendue en prenant en considération les points de vue des personnes qui participent à l'interaction et coproduisent de nouvelles spatialités (par exemple les acheteurs-ses de produits stupéfiants).

Comme évoqué au paragraphe précédent, les enjeux problématisés dans ces deux articles sont nombreux, et nos résultats invitent donc à poursuivre les recherches portant sur 
les microgéographies urbaines qui sont ainsi susceptibles d'être remodelées par de nouveaux dispositifs de surveillance par caméra vidéo et autres technologies.

Disponibilité des données. Les données quantitatives sousjacentes à ce document ne sont pas publiquement disponibles. Pour plus d'informations, veuillez contacter les auteurs.

Intérêts concurrents. Les auteurs déclarent qu'ils n'ont aucun conflit d'intérêts.

Remerciements. Les auteurs remercient les personnes suivantes pour leurs précieuse aide aux différentes étapes de cette étude : Noémie Béguelin Caudoux, Romaric Thiévent, Sarah Widmer, Geoffrey Klein, Bernard Taschini, André Faggioni, Didier Froideveaux, ainsi que les membres du groupe d'accompagnement mis en place pour le projet.

Edited by : Myriam Houssay-Holzschuch

Reviewed by : two anonymous referees

\section{Références}

Armanios, R. : Feu vert imminent pour la vidéosurveillance aux Pâquis, Le Courrier, Genève. 28.11.2013.

Armitage, R. : To CCTV or not to CCTV. A Review of Current Research into the Effectiveness of CCTV Systems in Reducing Crime, National Association for the Care and Resettlement of Offenders, London, 2002.

Bowers, K. J. et Johnson, S. D. : Measuring the geographical displacement and diffusion of benefit effects of crime prevention activity, J. Quant. Criminol., 19, 275-301, 2003.

Brown, B. : CCTV in Town Centres : Three Case Studies, Home Office, London, 1995.

Carli, V. : La vidéosurveillance est-elle un outil de sécurité et de gestion efficace pour lutter contre la criminalité, faire baisser le taux de criminalité et renforcer le sentiment de sécurité ?, Rapport de recherche, Centre international pour la prévention de la criminalité, Montréal, 2009.

Coleman, R. et Sim, J. : You'll never walk alone : CCTV surveillance, order and neo-liberal rule in Liverpool city centre, Brit. J. Sociol., 51, 623-639, 2000.

Département de la sécurité et de l'économie du Canton de Genève : La vidéoprotection devient réalité aux Pâquis, Communiqué de presse, 2014.

Ditton, J. et Short, E. : Yes, it works, no, it doesn't : Comparing the effects of open-street CCTV in two adjacent Scottish town centres, Crime Prev. Studies, 10, 371-403, 1999.

Fonteneau, M. : Vidéosurveillance et espaces publics : État des lieux des évaluations menées en France et à l'étranger, Institut d'Aménagement et d'Urbanisme, Paris, 2008.

Fyfe, N. R. et Bannister, J. : The Eyes Upon The Street, Images of the Street, Planning, Identity, and Control in Public Space, Home Office, London, 1998.
Gill, M. et Spriggs, A. : Assessing the impact of CCTV, Home Office, London, 2005.

Guéniat, O. et Klauser, F. : La vidéosurveillance : Quelle efficacité, l'Hebdo, 86-88, 2013.

Heilmann, E. : La vidéosurveillance, une réponse efficace à la criminalité ?, Criminologie, 36, 89-102, 2003.

Heilmann, E., Douillet, A. C., Dallaserra, J., Melchior, P. et Germain, S. : Vidéo-surveillance ou vidéo-protection, Le Muscadier, Paris, 2012.

Honess, T. et Charman, E. : Closed Circuit Television in Public Places : Its Accebtability and Perceived Effectiveness, Home Office, London, 1992.

Jeudy, H.-P. : Norme et transgression dans l'espace public, Espaces et Sociétés, 62-63, 83-91, 1990.

Kaenzig, R. et Klauser, F. : Evaluation de la vidéoprotection dans le quartier des Pâquis (2014-2016), Rapport de recherche, Université de Neuchâtel, Neuchâtel, accessible à l'adresse suivante : httpslunhboxlvoidb@x x lpenalty\@M ://www.unine.ch/ geographie/home/recherche/mandats-et-projets-acheves/paquis. html (la date du dernier accés : 10 février 2018), 2016.

Klauser, F. : Die Videoüberwachung öffentlicher Räume : zur Ambivalenz eines Instruments sozialer Kontrolle, Campus Verlag, Frankfurt am Main, 2006.

Klauser, F. and Kaenzig, R. : Territorialisation à distance par caméra vidéo : Perception de la vidéosurveillance au quartier des Pâquis à Genève, Geogr. Helv., 72, 271-282, https://doi.org/10.5194/gh72-271-2017, 2017.

Languin, I. : Vidéoprotection : dispositive efficace ou leurre sécuritaire ?, Tribune de Genève, 18.04.2013.

République et Canton de Genève : Politique commune en matière de lutte contre la criminalité 2014-2016, Convention signée le 17 septembre 2014, Genève, 2014.

Roselli, S. : Une vingtaine de caméras auront les Pâquis à l'œil. Tribune de Genève, 14.10.2014.

Sivarajasingam, V., Shepherd, J. P. et Matthews, K. : Effect of urban closed circuit television on assault injury and violence detection, Injury Prevention, 9, 312-316, 2003.

Skinns, D. : Crime reduction, diffusion and displacement : Evaluating the effectiveness of CCTV, in : Surveillance, Closed Circuit Television and Social Control, édité par : Norris, C., Moran, J. et Armstrong, G., Ashgate, Aldershot, 175-188, 1998.

Tilley, N. : Evaluating the effectiveness of CCTV schemes, in : Surveillance, Closed Circuit Television and Social Control, édité par : Norris, C., Moran, J. et Armstrong, G., Ashgate, Aldershot, 139-153, 1998.

Waples, S., Gill, M. et Fisher, P. : Does CCTV displace crime ?, Crimonology and Criminal Justice, 9, 207-224, 2009.

Welsh, B. C. et Farrington, D. P. : Crime Prevention Effects of Closed Circuit Television : A Systematic Review, Home Office, London, 2002.

Williams, K., Johnstone, C. et Goodwin, M. : CCTV surveillance in urban Britain : Beyond the rhetoric of crime prevention, in : Landscapes of Defence, édité par : Gold, J. et Revill, G., Prentice Hall, Harlow, 168-187, 2000. 